\title{
Anti-Ri Antibody Paraneoplastic Syndrome Without Opsoclonus-Myoclonus
}

\author{
Yadira Velazquez ${ }^{\mathrm{a}, \mathrm{b}}$, Divpreet Kaur ${ }^{\mathrm{a}}$, Aiesha Ahmed
}

\begin{abstract}
Anti-Ri antibody paraneoplastic neurologic syndrome historically has been linked to ataxia, opsoclonus-myoclonus and brainstem encephalitis, but these "cardinal features" are not necessary for its diagnosis, which should be considered in patients with subacute course of heterogeneous neurological manifestations. This is a report of a man who suffers from anti-Ri antibody paraneoplastic syndrome, with an atypical clinical presentation. His syndromic diagnosis was confirmed by serum western blot analysis that revealed the presence of anti-Ri antibodies. Clues to the underlying malignancy were given by FDG-PET scan that demonstrated a hypermetabolic peripancreatic lymph node. Besides highlighting clinical features of anti-Ri antibody syndrome that will help its early recognition and the appropriate steps for its diagnosis, we also discuss various treatment modalities that should be considered in this population in order to improve morbidity and mortality.
\end{abstract}

Keywords: Paraneoplastic syndromes; Nervous system; Anti-Ri antibodies; Opsoclonus-myoclonus syndrome

\section{Introduction}

Paraneoplastic syndromes affecting the nervous system are

Manuscript accepted for publication February 5, 2014

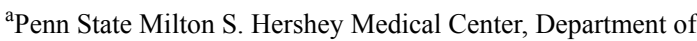
Neurology, 30 Hope Drive, P.O. Box 859, Hershey, PA 17033-0859, USA

${ }^{\mathrm{b}}$ Corresponding author: Yadira Velazquez, Penn State Milton S. Hershey Medical Center, Department of Neurology, 30 Hope Drive, P.O. Box 859, Hershey, PA 17033-0859, USA.

Email: dryadiravelazquez@gmail.com

doi: http://dx.doi.org/10.14740/jnr261w poorly understood disorders that cause significant morbidity and mortality in our patients. Well characterized antibodies associated to specific neurological syndromes in the presence of malignancy have been described, for example, the syndrome of ataxia and opsoclonus-myoclonus associated with anti-neuronal nuclear autoantibody 2 antibodies (antiRi or ANNA-2) first described by Dr. F. Antonio Luque in a patient with breast cancer [1]. However, in clinical practice the manifestation of a paraneoplastic-related nervous system disorder is frequently not through a well-defined syndrome like opsoclonus-myoclonus, limbic encephalitis or stiff person, but through a constellation of neurological signs and symptoms that involve multiple levels of the neuraxis, challenging its early recognition. Our case highlights an atypical presentation, challenges in diagnosis, clinical course and treatment response of anti-Ri antibody paraneoplastic syndrome.

\section{Case Report}

A 64-year-old right-handed man was transferred to our institution with progressive weakness and ventilator dependent respiratory failure. His disease started 3 months ago with fatigue, hypersomnia and progressive asymmetric lower extremity weakness. He also noticed diplopia, blurry vision, left eyelid ptosis and left eye abduction defect. One month later he had developed paraplegia in his legs and left arm paresis. He also noted dysarthria, hoarseness and dysphagia. He denied sensory symptoms, pain or cramps. His past medical history included diabetes mellitus and hypertension. His family history was unremarkable. He had smoked cigarettes for 40 years but quit 5 years ago.

On examination he was intubated and lethargic. He had intermittent tongue myoclonus, reactive pupils, bilateral horizontal gaze palsy and ptosis. Motor evaluation was characterized by atrophy in distal limbs, normal tone and no fasciculations. Motor weakness was found in the following muscles (MRC scale R/L): neck flexion 2, deltoids $4+/ 4+$, interossei $4+/ 4+$, finger extension $4+/ 4+$, hip flexion $3+/ 0$, knee extension $5 / 0$, knee flexion $4+/ 0$, ankle dorsiflexion $5 / 1$, ankle plantar flexion 5/1. Deep tendon reflexes were $(\mathrm{R} / \mathrm{L})$ : 
biceps $3+/ 3+$, triceps $3+/ 3+$, brachioradialis $3+/ 3+$, quadriceps $3+/ 2+$, achiles $2+/ 0$. Babinski sign was present bilaterally. There was no Hoffman sign or clonus. Sensation to light touch, pinprick and vibration was preserved throughout.

At our institution laboratory investigations included normal hematologic, comprehensive metabolic and thyroid panels. Gadolinium enhanced brain MRI and MRA of the head and neck were normal. Blood tests for Lyme disease, vitamins B1, B6, B12, zinc and copper levels were all normal. Muscle-specific tyrosine kinase receptor and acetylcholine receptor antibodies, including binding, blocking and modulating were unremarkable. Additional laboratory examinations demonstrated C-reactive protein of $2.33 \mathrm{mg} /$ $\mathrm{dL}$ (normal range: 0 - $1 \mathrm{mg} / \mathrm{dL}$ ), erythrocyte sedimentation rate of $43 \mathrm{~mm} / \mathrm{h}$ (normal: $1-17 \mathrm{~mm} / \mathrm{h}$ ), antinuclear antibody titer of $1: 160$ (normal: $\leq 1: 40$ ), negative rheumatoid factor, anti-thyroid peroxidase, anti-thyroglobulin antibodies, anti-neutrophil cytoplasmic, anti-dsDNA, anti-Smith, anti-Ro, anti-La, anti-Jo, anti-histone, anti-centromere and anti-SCL70 antibodies.

Nerve conduction studies were unrevealing. Repetitive nerve stimulation at $3 \mathrm{~Hz}$ of the left spinal accessory and ulnar nerves showed no abnormal decrement or increment in the compound muscle potential amplitude. Electromyogram revealed no active denervation in the axial, appendicular or bulbar musculature. Cerebrospinal fluid (CSF) demonstrated normal cell count and glucose, protein of $158 \mathrm{mg} /$ dL (normal: 18 - $58 \mathrm{mg} / \mathrm{dL}$ ). Unremarkable CSF parameters included: cryptococcus antigen, bacterial cultures, angiotensin converting enzyme level, venereal disease research laboratory test, herpes simplex I and II virus, toxoplasma, Epstein bar virus, cytomegalovirus antibodies and cytology examination for malignant cells.

His clinical presentation and ancillary tests suggested an autoimmune etiology and immunotherapy was administered, but five doses of methylprednisolone $1 \mathrm{~g} /$ day caused no functional improvement. Further blood tests revealed elevated CEA of $36.3 \mathrm{ng} / \mathrm{mL}$ (normal: 0 - $3 \mathrm{ng} / \mathrm{mL}$ ), normal prostate specific antigen, negative anti-Hu, anti-Yo antibodies and positive anti-Ri antibody titer of 1:640 (normal: not detectable). The diagnosis of paraneoplastic syndrome was evident and search for the underlying malignancy was undertaken. Meanwhile he received five cycles of plasmapheresis with moderate improvement in his left lower extremity strength, which now demonstrated antigravity strength from a previously plegic extremity.

Breast ultrasound and CT of the chest, abdomen and pelvis were obtained and unrevealing. FDG-PET scan demonstrated a $2.9 \times 2.5 \mathrm{~cm}$ hypermetabolic peripancreatic lymph node with elevated standardized uptake value of 13.3 suggestive of malignancy. Lymph node biopsy showed poorly differentiated carcinoma from pancreaticobiliary or pulmonary origin. The primary tumor has not yet been identified. Our patient is receiving intravenous cyclophosphamide with mild improvement.

\section{Discussion}

Anti-Ri antibodies historically have been linked to ataxia, opsoclonus-myoclonus and brainstem encephalitis, more often in females with ovarian or breast cancer. Although brainstem and cerebellar syndromes are the most frequent presentations, affecting respectively $71 \%$ and $50 \%$ of patients, a wide range of neurological signs and symptoms have been described. In a series of 28 anti-Ri positive patients $25 \%$ developed peripheral nervous system involvement, $21 \%$ cranial neuropathies, $18 \%$ myelopathy, $14 \%$ cortical involvement, $11 \%$ basal ganglia and neuromuscular junction dysfunction was seen in 4\% [2]. Our case highlights that the paraneoplastic syndrome associated with anti-Ri antibodies carries heterogeneous neurological manifestations and the absence of the "cardinal features" of opsoclonus-myoclonus, ataxia or brainstem encephalitis should not exclude the diagnosis. Anti-Ri associated paraneoplastic syndrome should be suspected in the presence of subacute multifocal neurological manifestations.

For the diagnosis, CSF evaluation and neuroimaging should be obtained but it reveals non-specific abnormalities in most cases: CSF analysis shows proteinorraquia in $37 \%$ of patients, mild lymphocytosis in $42 \%$ and negative cytology in $100 \%$. Imaging of the central nervous system may be normal or demonstrate T2 hyperintensities, parenchymal, cauda equina or root gadolinium enhancement [1]. Confirmatory testing will depend on western blot analysis of the patient's serum [3].

Anti-Ri antibodies have been linked to small cell lung carcinoma and gynecologic malignancy; however, nonsmall cell lung cancer, bladder carcinoma [2], transitional cell carcinoma of the renal pelvis or thymus [4], melanoma and malignant fibrous histiocytoma have also been described [5]. In some cases the diagnosis of cancer has been remote, with variable recurrence at the time of neurological presentation. In others no cancer has been found or the underlying malignancy was discovered many years after the initial neurological manifestations [2]. Routine tumor screen should include mammogram, gynecologic examination, testicular ultrasound, high resolution $\mathrm{CT}$ of the chest, and if these are negative, FDG-PET should be obtained. As evidenced in our patient biopsy of small lesions such as adenopathies may confirm an underlying malignancy and should be performed. Continued surveillance for many years is necessary for cancer detection if the initial cancer screening was unrevealing [5].

Regarding treatment, tumor-directed therapy is the main intervention, although administration of immunosuppressive therapy has been suggested in those patients without identified malignancy, in whom some degree of neurological re- 
covery may be possible. In the cohort described by Pittock, $60 \%$ of patients with paraneoplastic neurological syndromes developed wheelchair dependence, and most of them demonstrated neurological recovery after tumor-targeted radiation or immunosuppressive therapy with steroids, plasmapharesis or intravenous immunoglobulin therapy, alone or in combination [2]. With treatment there appears to be a decrement in the anti-Ri antibody titter [2], but at this moment it is still unclear if this could be used as a reliable predictor of remission and long term prognosis.

\section{Conclusions}

Anti-Ri antibody associated paraneoplastic syndrome has been linked to ataxia, opsoclonus-myoclonus and brainstem encephalitis, but frequently its neurological manifestations go beyond those cardinal signs. Its diagnosis should be thought in all those patients with subacute heterogeneous neurological manifestations. Cancer detection and treatment is the mainstream of management. Immune therapy should be offered to those patients without identified malignancy to improve morbidity. Follow-up and malignancy screening should continue for at least 5 years in order to diagnose an occult malignancy and offer tumor-specific therapy.

\section{References}

1. Luque FA, Furneaux HM, Ferziger R, Rosenblum MK, Wray SH, Schold SC, Jr., Glantz MJ, et al. Anti-Ri: an antibody associated with paraneoplastic opsoclonus and breast cancer. Ann Neurol. 1991;29(3):241-251.

2. Pittock SJ, Lucchinetti CF, Lennon VA. Anti-neuronal nuclear autoantibody type 2 : paraneoplastic accompaniments. Ann Neurol. 2003;53(5):580-587.

3. Moll JW, Antoine JC, Brashear HR, Delattre J, Drlicek M, Dropcho EJ, Giometto B, et al. Guidelines on the detection of paraneoplastic anti-neuronal-specific antibodies: report from the Workshop to the Fourth Meeting of the International Society of Neuro-Immunology on paraneoplastic neurological disease, held October 2223, 1994, in Rotterdam, The Netherlands. Neurology. 1995;45(10):1937-1941.

4. Sutton IJ, Barnett MH, Watson JD, Ell JJ, Dalmau J. Paraneoplastic brainstem encephalitis and anti-Ri antibodies. J Neurol. 2002;249(11):1597-1598.

5. Vedeler CA, Antoine JC, Giometto B, Graus F, Grisold W, Hart IK, Honnorat J, et al. Management of paraneoplastic neurological syndromes: report of an EFNS Task Force. Eur J Neurol. 2006;13(7):682-690. 